\title{
Mopping up a molecule for breast cancer survival
}

Many cancers suppress immune surveillance to avoid an antitumour response. However, scientists have reported in Nature Immunology that breast cancer cells promote immune cell infiltration, and that infiltrating myeloid cells can be usurped to promote breast cancer cell survival. Thymic stromal lymphopoietin (TSLP) produced by myeloid cells promoted breast cancer cell survival, and anti-TSLP antibodies prevented primary tumour growth and metastasis to the lungs in mouse models.

The role of TSLP in cancer has been controversial. This cytokine promotes an inflammatory immune response, and is associated with a good or poor prognosis in different tumour types. To examine TSLP in breast cancer, the authors used CRISPRCas to remove Tslp from the oncogenic 4T1 mouse cell line. TSLPdeficient $4 \mathrm{~T} 1$ cells had lower survival in culture, but in transplantation

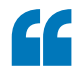

intranasal administration of an antiTSLP antibody reduced metastases

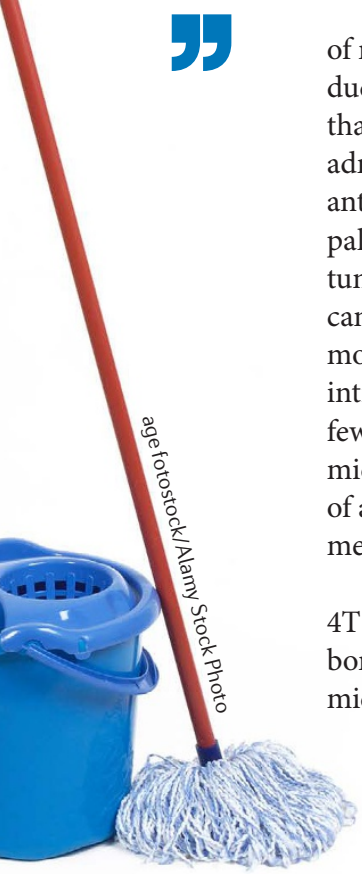

experiments the primary tumour was the same size as tumours derived from the parental line, suggesting that a non-tumour source of TSLP could promote cancer cell survival in vivo. In support of these data they found that 4T1 cells engineered to lack the TSLP receptor also failed to generate tumours in wild-type mice.

Additional data support the role of non-tumour TSLP. 4T1 cells produced smaller tumours in Tsl $p^{-/-}$mice than in wild-type mice. Systemic administration of an anti-TSLP antibody, begun when tumours were palpable, reduced the growth of tumours in another model of breast cancer, the MMTV-PyMT transgenic mouse. When $4 \mathrm{~T} 1$ cells were injected intravenously, $T s l p^{-/}$mice developed fewer metastases than did wild-type mice, and intranasal administration of an anti-TSLP antibody reduced metastases in wild-type mice.

To identify the source of TSLP, $4 \mathrm{~T} 1$ cells were transplanted into bone marrow chimeras. Wild-type mice reconstituted with $T s l p^{-/}$bone marrow had smaller 4T1-derived tumours than $T s l p^{-/}$mice reconstituted with wild-type bone marrow, suggesting that haematopoietic cells are key TSLP producers. Neutrophils seem to be a particularly important source; transplantation of neutrophils from tumour-bearing wild-type mice could restore the size of the primary tumour in Tsl $p^{-/}$mice.

Interleukin (IL)-1 $\alpha$ is expressed in human breast tumours, and induces TSLP expression in immune cells. 4T1 cells deficient in IL1a generated smaller tumours than did wild-type 4T1 cells, and neutrophils isolated from tumour-bearing mice produced TSLP in response to IL-1a stimulation ex vivo. These results support a model in which breast cancer cells recruit myeloid cells through IL-1 $\alpha$, and the infiltrating neutrophils then promote cancer cell survival by secreting TSLP.

Anti-TSLP antibodies are being investigated as potential therapies for asthma and allergy; these antibodies could also be useful in treating primary or metastatic breast cancer.

Megan Cully

ORIGINAL ARTICLE Kuan, E. L. \& Ziegler, S. F. A tumor-myeloid cell axis, mediated via the cytokines IL-1 $\alpha$ and TSLP, promotes the progression of breast cancer. Nat. Immunol. 19, 366-374 (2018) 